\title{
Immersive storytelling in mixed reality environments
}

\author{
Denise Doyle \\ Faculty of Arts \\ University of Wolverhampton, UK \\ D.Doyle@wlv.ac.uk
}

\begin{abstract}
How will we adapt to a future that may see humans as an interplanetary species? The proposed project uses themes of outer space, future worlds and space travel to examine ways in which our future identities may be formed from these new environments, the role/s we may have in future societies; and the relationships that we will form with the people we will meet. The utilization of virtual and mixed reality $(A R / V R)$ technologies can be a powerful tool in which to place the audience in different scenarios, to experience it from different viewpoints, and to allow them to anticipate what the future may look, feel like, and indeed be like, by being placed into a set of future space scenarios. This paper presents ideas from an interdisciplinary team of artists, scientists, and technologists of methodological approaches for art-science-technology and the prototypes anticipated through these dialogues.
\end{abstract}

Keywords-mixed reality; immersive storytelling; VR/AR; artscience methods; artist residencies

\section{INTRODUCTION}

"Artists can challenge prevailing ideologies and imagine alternative worlds, and for this reason art plays a vital role in imagining our space futures, ${ }^{[1]}$.

A recent UK government report concluded that people's identities are, and will continue to be, significantly affected by several drivers of change and "in particular the rapid pace of developments in technology" and further suggests that the internet has been "instrumental in raising awareness that identities are more multiple, culturally contingent and contextual than has previously been understood" ${ }^{[2]}$.

Despite the proliferation of art/science/technology initiatives around the world, there have been few attempts to articulate a set of methodologies for collaborative art and science research processes ${ }^{[3]}$. Artists and scientists can approach the question of identity and embodied experience in the context of new technologies in different ways. Neuroscientists Emily Cross and Luca Ticini suggest that 'art, like everything else we do, is generated from electrical impulses passed between the synapses of the brain, expressed through the body, and eventually appreciated through the senses' [4], whilst Arts Director Sian Ede writes that "we so sensuously inhabit our bodies that it is hard to see them as systems of knowledge, even in the purified arena of the laboratory or operating table' [5]
The Digital Agenda for Europe and the Human Brain Project aims to better understand and improve our knowledge of the brain and its working processes, and through advancing research in the field of neuroscience a number of studies now show a developing understanding of the level of the plasticity of the brain itself ${ }^{[6]}$. There have been studies of how the brain reacts in digital space demonstrating significant effects on the emotional, psychological, perceptual experience of the participants ${ }^{[7]}$. Other studies have focused on the experience of the body and the self in digital space, and on concepts of identity in relation to the digital self. These advances in neuroscience, body sensor monitoring, multi-sensory perceptual understandings, and how people can be psychologically enhanced by such experiences are opening up new ways of creating meaningful virtual experiences.

The conceptual foundations of interacting with immersive mixed reality environments were already being laid at the end of the previous century through the work of artist Char Davis ${ }^{[8]}$, and dancers and choreographers explored new embodied experiences in weightlessness through zero gravity flight conditions ${ }^{[9]}$. Twenty years on with a whole new generation of accessible technologies such as the VR Oculus Rift, HTC Vive, and other technologies that enable augmented or mixed reality experiences such as upcoming wearables and see-through headsets (HoloLens) there is a need to increase the cultural, social, and phenomenal understanding of these new spaces and accessible worlds and to develop positive and enriching experiences for the individual and society as a whole. Initial applications of mixed and augmented realities are now being applied to the cultural, economic, and political experiences of global audiences in live performance cultural contexts (Golem, 1927 Group, 2015; Theatre for Robots Only, 2015; Bjork Digital, 2016; The Tempest, Royal Shakespeare Company, 2017) and virtual museum contexts (At Home in the Cosmos, 2007; Microscopia, 2014; Viking, 2015; Thresholds, 2017).

\section{MIXING REALITIES}

After having playfully enjoyed an early AR art installation presented at ISEA 2009 in Belfast entitled 52 Card Psycho (2009) where 52 cards, each printed with a unique identifier, play video clips of shots from the film, two recent technology mediated experiences have challenged my previous assumptions of the possibilities around VR and AR. Firstly an immersive experience at the Bjork Digital exhibition and her work entitled Notget VR (2016) that of an encounter with a morphing and slowly growing 'avatar' of Bjork in an extraordinary VR experience using HTC Vive. Bjork writes of her use of VR that it is "not only a continuity to the music video but has an even more theatrical potential, ideal for this emotional journey" ${ }^{[10]}$. This experience has remained with me as an intensely altered sense of reality, intensified by the sense of immersion and immediacy possible in total VR. Secondly watching The Tempest at the Royal Shakespeare Company in 
Stratford, UK in late 2016 where a whole range of techniques including live performance capture by the character and actor Ariel were employed to attempt to integrate new technologies in a live performance context - this gave a glimpse of what could be possible in the very near future. Regarded as one of Shakespeare's most magical of plays, and having already inspired filmmaker Peter Greenaway to push the boundaries of new technologies with an early use of mixed reality techniques in the 1990s in Properos Books (1993) the impact of having 'moments' of the performance enhanced by technology was enough to convince me that more was possible. VR and mixed reality hold the potential of a new set of creative and emotional experiences beyond our current perception.

However, the challenges right now consist in developing concepts for interaction design and immersive storytelling, taking full advantage of the technology available and making them intuitively usable in different application scenarios. The technology required to actually implement these immersive mixed reality environments is only now becoming mature enough for real-world applications to provide a more satisfying user experience. Whereas previous solutions suffered from low frame rates, high latencies and exorbitant costs, current HeadMounted Displays (VR Oculus Rift, HTC Vive) and Smartglasses (Microsoft HoloLens) are already "good enough" for many applications and will further improve over the coming years ${ }^{[11]}$.

Other research highlights the storytelling challenges in Virtual Reality ${ }^{[12]}$ and focuses on new approaches in developing a language of storytelling for immersive VR (based on attention and engagement). Interacting in a fully immersive VR space may require different storytelling strategies to those required for augmented mixed reality where the user is interpreting information from the physical and virtual world at the same time, or creating narratives for robots, humans, and avatars may require other narrative techniques. There is a need to test narrative concepts for a range of mixed reality (VR/AR) scenarios.

\section{METHODOLOGICAL APPROACH}

Gunter H. Oettinger, the Commissioner in charge of the Digital Single Market, suggestion is "in the age of digitisation art and engineering are no longer contradictory modes of thinking" ${ }^{[13]}$. This is acknowledged in the development of the new European initiative STARTS programme, and evidenced by the outcomes of the ICT ART CONNECT programme of 2013 and 2014. The proposal responds to the spirit of this new era in its development of new modes of research that cross disciplines and bridge especially Art and Science disciplines. We are on the verge of a new era in expanded human development that augmented and virtual realities will support; we need diverse teams to make this all it can be. The project is interdisciplinary at its core, joining qualitative and quantitative approaches from the arts and sciences during the implementation of the proposed activities (eg. using mixed methods including interviews and narrative analysis, reflective research practice, and the analysis of data (action and gesture) during embodied interaction.
A range of activities are proposed and in particular the engagement of artists with the central topic of the proposal, that of the future impact of technology and our anticipated futures. Two sets of artist residencies are anticipated: (i) at SPECS Research Lab in Barcelona and the artists will engage with the nature of living robots, embodied interactions in mixed reality and live performance; (ii) at the European Astronaut Centre in Cologne. The artists will engage in the study of embodiment in space and narratives of space travel. The SPECS research lab and the EAC training facility were chosen for the artist residences specifically to enable artists to consider the wider implications of living with technology over the next half century. SPECS Laboratory uses synthetic methods to study and synthesize the neuronal, psychological and behavioural principles underlying perception, emotion and cognition. Since 1998 Verschure and his co-workers have generalized the basic science towards complex real-world installations and exhibits and have constructed over 20 prototype interactive multi-media systems ${ }^{[14]}$. The EAC is an establishment of the European Space Agency (ESA) and in charge of the training and medical support of astronauts to the International Space Station (ISS). Training facilities for ISS astronauts include mock-ups of ESA elements at the ISS, virtual reality training tools, Neutral Buoyancy Facility (NBF) to simulate micro-gravity aspects for training of extravehicular activities; control rooms and simulation facilities. At the astronauts training facility the artists will have access to the new lunar training analogue facility LUNA, European Exploration Lab $\left(900 \mathrm{~m}^{2}\right)$ due to be operational by spring 2018.

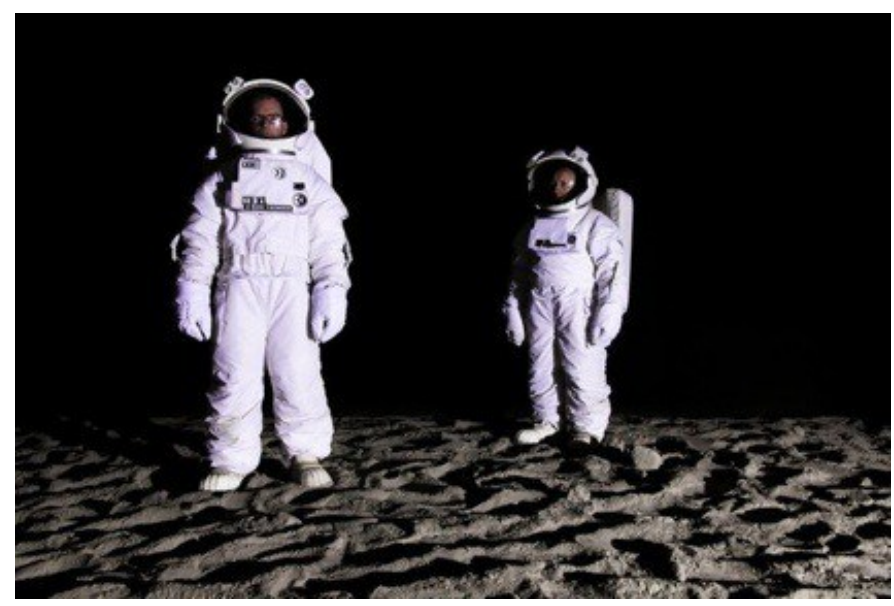

Figure. 1. Bored Astronauts on the Moon, Harrison \& Wood, 2011 C Harrison \& Wood (Courtesy of the Artists)

In addition to the artist residencies we will conduct astronaut interviews to enable us to study the psychology of remote environments and we will gather first-hand accounts of male $\&$ female astronauts and their experience of inhabiting outer space through interview and analysis. We will research the psychology of remoteness ${ }^{[15]}$ and how outer space can challenge traditional notions of gendered space ${ }^{[16]}$.

As part of the art-science activities outlined we will utilise the eXperience Induction Machine (XIM) facility at SPECS, a 
multimodal mixed reality space. This enables human behavior to be analyzed and in particular the study of embodied interaction in mixed reality space (see Fig. 2 and Fig. 3 below).

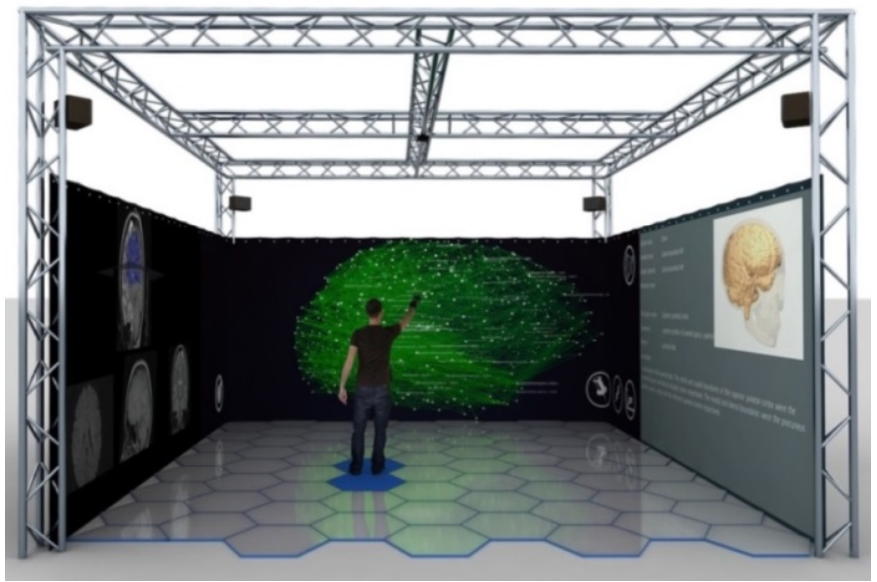

Figure. 2. The eXperience Induction Machine XIM. A multimodal mixed reality space at SPECS research lab. Brainx3.com (C) SPECS

Using this space we will evaluate the type of actions and gestures performed in interactive $\&$ immersive mixed reality environments. Using motion capture technology (Kinect) \& physiological sensors (heart rate, EDR, breathing), we will quantify how cognitive and emotional processes (spatial memory and appraisal) are influenced by navigating in 3 types of mixed reality space: XIM; VR (HTC Vive); AR (HoloLens). The results will inform the development of the prototype concepts.

The originality of this research methodology lies (a) in the embedding of the artists within the ICT innovation process, and (b) the novel approaches to the engagement of audiences through mixed reality (VR/AR) technologies using gamified storytelling features and digital performance with robots, humans and avatars.

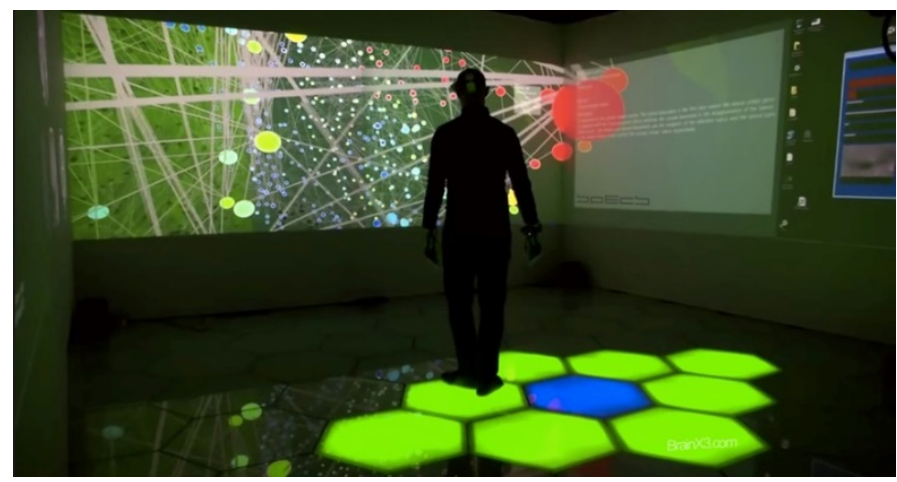

Figure. 3. User during a discovery session in the eXperience Induction Machine XIM. A multimodal mixed reality space at SPECS research lab. (C) SPECS

\section{IMMERSIVE STORYTELLING MODES}

The utilization of virtual and mixed reality (AR/VR) technologies can be a powerful tool in which to place the audience in different scenarios, allow them to anticipate what the future may look and be like in order to explore ideas of how future space exploration may impact on our future identity. In the project we propose to engage in different forms of mixed reality, VR and AR, and live performance (see Fig. 4 below). The project aims to develop three interactive prototypes (installation, book/s, and performance) that investigate "future identity" as it is shaped by our environment, our society, and our social interactions and: introduce children to "jobs of the future" and allows them to understand the skills and training that will be required to practice each job; engage young people in the significance of "living with robots" and other sentient technologies; and enable young audiences to explore a number of future space exploration scenarios.

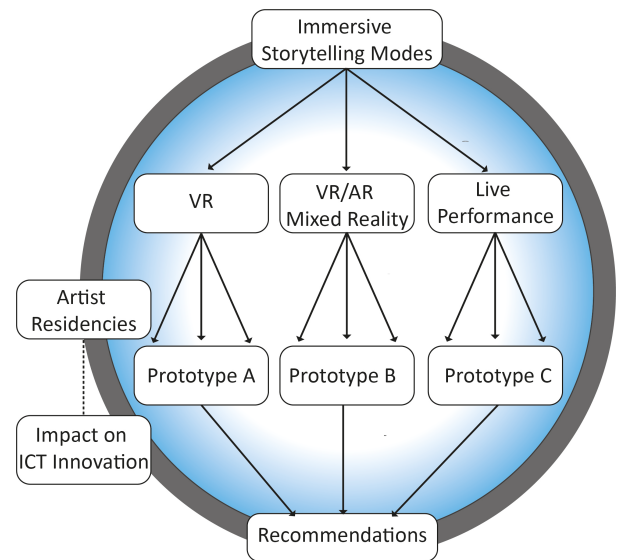

Figure 4: Immersive Storytelling Modes (C) Denise Doyle

The three prototype concepts are:

\section{Prototype 1:AR/VR Interactive Installation}

The future of identity can be related to several efforts to reach Mars, or asteroids, or a return to the Moon, and in anticipating and imagining other alternative worlds. How does our environment shape our identity? VR technologies, and virtual world technologies are being utilized in preparation for further exploration of space travel but how do these efforts change our internal and intrinsic view of our self that inhabits both the physical and the virtual with fluid and interconnected identities? It raises the question: What will our identities be like thirty years, or even fifty years from now? Using VR technologies, with multi-user options, and an integrated tracked virtual reality system, the prototype will develop a series of three immersive narratives in which young adults (16-19 years) can explore our future interplanetary existence on the Moon, Mars, and Earth. What will these new environments be like, who will we meet in them, and how will that shape our future identities? By completely immersing the audience in the scenario, those questions may be encountered as more immediate: what would life on Mars be like; what would an interactive encounter with aliens entail; if gender is so bound 
up with nature on Earth will leaving it enable us to perceive gender in more fluid ways ${ }^{[16]}$.

\section{Prototype 2:Mixed Reality Interactive Book/s}

Using mixed (augmented and virtual) reality technologies and gamified storytelling features this prototype aims to introduce children to the "jobs of the future" in a time when (wo)man may inhabit the moon. Employing analogies of past and present jobs, the prototype will present different possibilities and environments that will allow children to understand complex concepts such as the skills and training that will be required to practice each job. In what ways can mixed realities impact on our understanding of future work scenarios? The prototype will comprise of a series of short books and interactive cards that will use AR/VR technologies to explore future work possibilities, such as a lunar miner working on the moon base station, or a Martian architect. Using the interactive capabilities provided by these technologies children will be able to identify themselves with the characters appearing during the activities; explore their future identities and roles in future societies; and choose the interactive story they want to 'live' (making the story for each child different). The prototype will also include the HoloDeck4D technology developed by partner, CTIC, as well as other VR devices (e.g. Oculus Rift, Cardboard). The prototype is aimed at 7-11 year olds and will also explore how "the social imaginary of outer space, [is] more than just a collection of ideas, [it] helps to shape society's values" ${ }^{[1]}$. and will incorporate discussions about old colonization on earth.

\section{Prototype 3:A Human-Machine Symbiotic (HMS) Performnce}

Living with Robots and the theme of changing identities in networked space are developed in this prototype. Current research points "to an emerging trend towards hyperconnectivity" and highlights changing forms of identity from an internal, subjective self to a less robust external, networked self ${ }^{[2]}$. With robots gaining "personhood" status, there will be a new set of ethics and realities of living and working with robots. Understanding these will be the key to the success of living with sentient technologies. This experimental performance will explore the confluence of the physical and the virtual dimensions underlying existence and experience. For the performance we will stage human and virtual actors (dancers, avatars, robots) that will interact in a future world scenario and explore the significance of artificial sentience and the ability of humans to create and co-exist with it. The prototype performance is aimed at a diverse audience including young adults (16-25 years) and uses novel means of interactive performance that shows and mixes two co-dependent worlds (the real and the virtual) in order to create a mixed experiential space $^{[17]}$.

\section{CONCLUSION}

More than ten collaborators across Europe and beyond have come together to develop this speculative proposal. We hope in the next two years to be able to develop and realize the research and the prototypes described above. I recently attended an induction day at my four year old daughter's school in which the Head Teacher confidently explained that she and her staff are preparing and educating children for jobs that do not yet exist, in a world that has and is going to be transformed even further by the 'future of technology'. We hope that engaging in the future of technology in ways described above may go some way to preparing audiences, and in particular younger audiences, to prepare themselves for the challenges that they may face in the future, and how they may be able to direct the future a little more as they engage in what will inevitably become an even more technological and complex world.

\section{REFERENCES}

[1] Triscott, N. (2016) Transmissions from the Noosphere: Contemporary Art and Outer Space, in Ormrod, J.S. \& Dickens, P. The Palgrave Book of Society, Culture and Outer Space, Palgrave Macmillan UK

[2] Future Identities: Changing Identities in the UK, UK Government Report, 2013, https://www.gov.uk/government/publications/futureidentities-changing-identities-in-the-uk

[3] Malina, R. F. (2011) Alt.Art-Sci: We Need New Ways of Linking Arts and Sciences. Leonardo, Vol. 44.1.

[4] Cross, E. S. \& Ticini, L. F. (2012). Neuroaesthetics and beyond: New horizons in applying the science of the brain to the art of dance. Phenomenology and the Cognitive Sciences, 11(1), 5-16.

[5] Ede, S. (2005). Art and Science, I.B. Taurus Publishing.

[6] Greenfield, S. (2014). Mind Change: How digital technologies are leaving their mark on our brains, Rider Publishing.

[7] Morie, J. F., Haynes, E., and Chance, E. (2011) Warriors' Journey: A Path to Healing through Narrative Exploration. International Journal on Disability and Human Development. 10 (1).

[8] Davies, C. (1995) Osmose, http://www.immersence.com/osmose/

[9] Bureaud, A. \& Dubois, K. (2005) "The Embodiment of (Micro)Gravity. Kitsou Dubois's Analogies: An Artistic and Aesthetic Experience" In Space: Planetary Consciousness and the Arts Symposium Proceedings. Yverdon-les-Bains, Switzerland; Doyle, D. (2015) Out of this World: Exploring Embodiment and Space through Artistic Processes and Practice, International Journal of Performance Arts and Digital Media, 10.1, pp. 1-18, Routledge.

[10] Bjork (2017). See http://bjorkdigital.cccb.org/en/

[11] Heim, M. R. (2017) Virtual Reality Wave 3, In Gackenbach, J \& Bown, J. (Eds.) Boundaries of Self and Reality Online: Implications of Digitally Constructed Realities. Elsevier, Cambridge Massachusetts.

[12] Brillhart, J. (2016) In the Blink of a Mind, https://medium.com/thelanguage-of-vr/tagged/editing

[13] See European Commission (2016c) STARTS Programme, https://ec.europa.eu/digital-single-market/en/ict-art-starts-platformSee SPECS http://specs.upf.edu/installations

[14] Wu et al 2015, 2016; Morie, J.F., et al (2011) Operational Assessment Recommendations: Current Potential and Advanced Research Directions for Virtual Worlds as Long-Duration Space Flight Countermeasures, NASA/TP-2011-216164; Patterson, C. (2016) Interstellar Messaging: An Embodied Perspective, In Campion, N. (Ed) Heavenly Discourses, Sophia Centre Press, University of Wales.

[15] Deerfield, K. (2016) Heavenly Bodies: Gender and Sexuality in ExtraTerrestrial Culture. PhD Cardiff University. Unpublished.

[16] RE-FLUX (2016) Multi-modal performance with AI, robots, VR and Humans http://specs.upf.edu/installation/3162 